\title{
Expression of Pink1 with $\alpha$-synuclein in the dopaminergic neurons of Drosophila leads to increases in both lifespan and healthspan
}

\author{
A.M. Todd and B.E. Staveley \\ Department of Biology, Memorial University of Newfoundland, \\ St. John's, Newfoundland and Labrador, Canada \\ Corresponding author: B.E. Staveley \\ E-mail: bestave@mun.ca \\ Genet. Mol. Res. 11 (2): 1497-1502 (2012) \\ Received July 4, 2011 \\ Accepted February 16, 2012 \\ Published May 21, 2012 \\ DOI http://dx.doi.org/10.4238/2012.May.21.6
}

\begin{abstract}
Overexpression of the gene coding for $\alpha$-synuclein has been shown to be an inherited cause of Parkinson disease. Our laboratory has previously co-expressed the parkin and Pinkl genes to rescue $\alpha$-synuclein-induced phenotypes within a Drosophila model. To further investigate the effect of Pink1 in this model, we performed longevity and behavioral studies using several drivers to express the $\alpha$-synuclein and Pink1 genes. Our findings showed that overexpression of Pink1 and overexpression of Pinkl with $\alpha$-synuclein resulted in an increased lifespan when driven with the TH-Gal4 transgene. This increase in longevity was accompanied by an increased healthspan, as measured by mobility over time, suggesting that this is an example of improved functional aging. Our results indicate that, in the dopaminergic cells targeted by TH-Gal4, increased expression of $\alpha$-synuclein and Pink1 together have a synergistic effect, allowing for enhanced protection and increased survival of the organism.
\end{abstract}

Key words: Pink1; $\alpha$-synuclein; TH-Gal4; Drosophila; Longevity; Healthspan 


\section{INTRODUCTION}

Parkinson disease (PD) affects 1-2\% of the population over the age of 65 years, where age is the largest risk factor for the development and progression of the disease (Lees et al., 2009). PD is characterized by a progressive loss of dopaminergic neurons in the substantia nigra pars compacta, often including the presence of ubiquitin-positive and $\alpha$-synucleinenriched inclusions, known as Lewy bodies, in the remaining neurons. Several genes have been linked to familial forms of PD, including the genes coding for $\alpha$-synuclein and PTENinduced putative kinase 1 (PINK1).

Identified as a kinase, PINK1 has been shown to locate to the mitochondria and is hypothesized to be involved in their protection (Deas et al., 2009). Mutations in PINK1, and in the Drosophila homologue Pink1, show substantial mitochondrial defects in sensitive tissues, with the inability to inhibit cytochrome c release under stress conditions (Clark et al., 2006; Park et al., 2006; Yang et al., 2006; Exner et al., 2007; Wang et al., 2007). As a protective protein, PINK1 may confer protection of the mitochondria through several mechanisms (Deas et al., 2009), including an interaction with molecular chaperones to regulate oxidative stress responses, activation of the parkin E3 ubiquitin ligase to result in the tagging of toxic proteins, such as $\alpha$-synuclein, for degradation, or through the initiation of fission events to remove dysfunctional mitochondria via mitophagy.

Overexpression of the gene encoding $\alpha$-synuclein has been shown to be an inherited cause of PD, and a transgenic $\alpha$-synuclein-induced model in Drosophila has been successfully used to mimic the degenerative processes seen in PD (Feany and Bender, 2000; Whitworth et al., 2006). Previous study in our laboratory has shown the ability of parkin overexpression and Pinkl overexpression to rescue an $\alpha$-synuclein-induced PD-like phenotype in Drosophila melanogaster, presumably through the targeting of the $\alpha$-synuclein protein for degradation (Haywood and Staveley, 2004; Todd and Staveley, 2008). To further investigate the effect of Pinkl in this model, we performed longevity and behavioral studies using several neuronal and ubiquitous drivers to express the $\alpha$-synuclein and Pink1 transgenes.

\section{MATERIAL AND METHODS}

Dr. M. Feany (Harvard Medical School) generously provided the UAS- $\alpha$-synuclein flies (Feany and Bender, 2000) and Dr. J. Hirsh (University of Virginia) provided the Dopa decarboxylase-Gal4 (Ddc-Gal4) flies (Li et al., 2000) and the tyrosine hydroxylase-Gal4 (TH-Gal4) flies (Friggi-Grelin et al., 2003). The UAS-Pink1 transgenic line was created using the GH20931 D. melanogaster Pink1 clone (Todd and Staveley, 2008). The UAS- $\alpha$-synuclein;UAS-Pink1 line was generated using standard techniques. The $w^{1118}$ flies were obtained from Dr. Howard Lipshitz at the Hospital for Sick Children in Toronto. The GawB ${ }^{C 739}$-Gal4, GawB ${ }^{V 55}$-Gal4, Elav-Gal4, Arm-Gal4, and UAS-GFP were obtained from the Bloomington Drosophila Stock Center at Indiana University. All flies were cultured on standard cornmeal $/$ yeast $/$ molasses/agar medium at $25^{\circ} \mathrm{C}$.

Two hundred adult males of each genotype were collected under gaseous carbon dioxide and aged upon standard cornmeal/yeast/molasses/agar medium, at $25^{\circ} \mathrm{C}$, in upright standard plastic shell vials. Flies were maintained in non-crowded conditions with one to twenty individuals per vial. Flies were scored for viability every two days and transferred to fresh medium 
according to an established protocol (Staveley et al., 1990). Forty adult males of each genotype were assayed for climbing ability (Todd and Staveley, 2004). Flies were maintained on standard cornmeal/yeast/molasses/agar medium at $25^{\circ} \mathrm{C}$ and were assayed every seven days.

\section{RESULTS}

Overexpression of Pinkl increases lifespan when driven with TH-Gal4 (Figure 1A). In addition, overexpression of Pink1 with $\alpha$-synuclein, using the TH-Gal4 driver, results in a dramatic increase in lifespan. The results suggested that increases in expression of $\alpha$-synuclein and Pink1 together can have a synergistic effect, allowing for enhanced cellular protection and increased survival. When assessing the extension of lifespan observed in Figure 1A, it was necessary to determine if this was accompanied by improved healthspan or functional aging in the surviving individuals. D. melanogaster exhibit a strong negative geotactic climbing response, allowing for the conduct of mobility assays within the $\alpha$-synuclein-induced model (Haywood and Staveley, 2004; Todd and Staveley, 2008). When we assessed the climbing ability of flies expressing Pink1, using the TH-Gal4 driver (Figure 1B), there was a rescue of the characteristic $\alpha$-synuclein-induced phenotype of premature loss of climbing ability. In addition, flies expressing Pinkl with $\alpha$-synuclein using the TH-Gal4 driver had a significant increase in climbing ability in the surviving flies as compared to other genotypes of the same age. This suggests that the increase in longevity was an example of healthy aging, and that the lifespan difference observed between Pinkl with $\alpha$-synuclein and Pinkl expression alone was a synergistic effect.

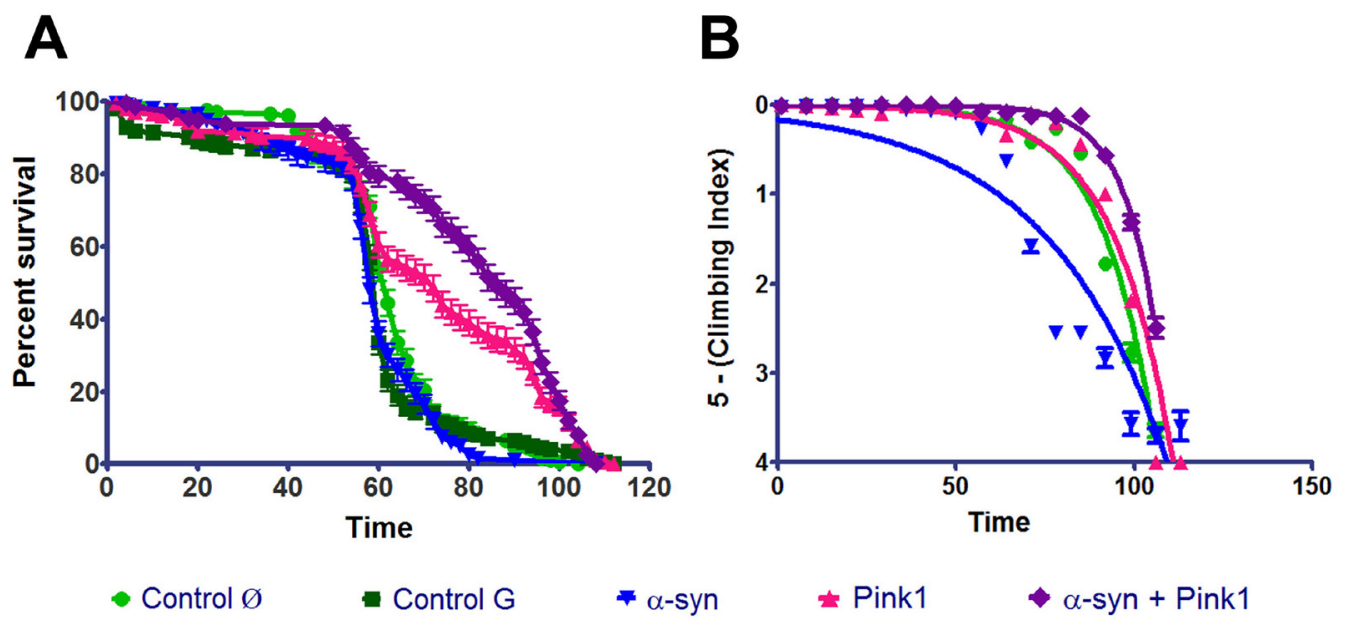

Figure 1. Effect of Pink1 and $\alpha$-synuclein expression on longevity (A) and mobility (B) when driven with TH-Gal4. Overexpression of Pinkl using the TH-Gal4 driver shows an increase in lifespan (mean $=72$ days) as compared to controls (mean $=61 \pm 1$ days), $\mathrm{P}<0.0001$ (A). Overexpression of Pink1 with $\alpha$-synuclein using the TH-Gal4 driver shows a dramatic increase in lifespan (mean $=86$ days) as compared to controls (mean $=61 \pm 1$ days), $\mathrm{P}<$ 0.0001. Overexpression of Pink1 results in a rescue of the $\alpha$-synuclein-induced premature loss of climbing ability when driven with TH-Gal4 (B). Overexpression of Pink1 with $\alpha$-synuclein, using TH-Gal4, shows significantly increased climbing ability above that of controls (non-linear curve fit comparison, $C I=95 \%$ ). Survival curves were compared using the log-rank test. Genotypes expressed include $w^{1118}$ (Control Ø); UAS-GFP/+ (Control G); UAS$\alpha$-synuclein $/+(\alpha$-syn); UAS-Pink1/+ (Pink1), and UAS- $\alpha$-synuclein $/+; U A S$-Pink1/+ $(\alpha$-syn + Pink1). Error bars indicate standard error of the mean. 
To investigate the effect of $\alpha$-synuclein and Pink1 expression in other cell populations, longevity studies were performed using a variety of established constitutive drivers (Figure 2). The significant increases in lifespan shown with $T H$-Gal4 (Figure 1A) were not observed when using other drivers, including neuronal (Elav-Gal4, GawB ${ }^{C 739}$-Gal4, GawB $B^{V 55}$-Gal4) and ubiquitous (Arm-Gal4) drivers. This suggests that the increases in longevity were dependent on expression within the dopaminergic neurons targeted by $T H$-Gal4.
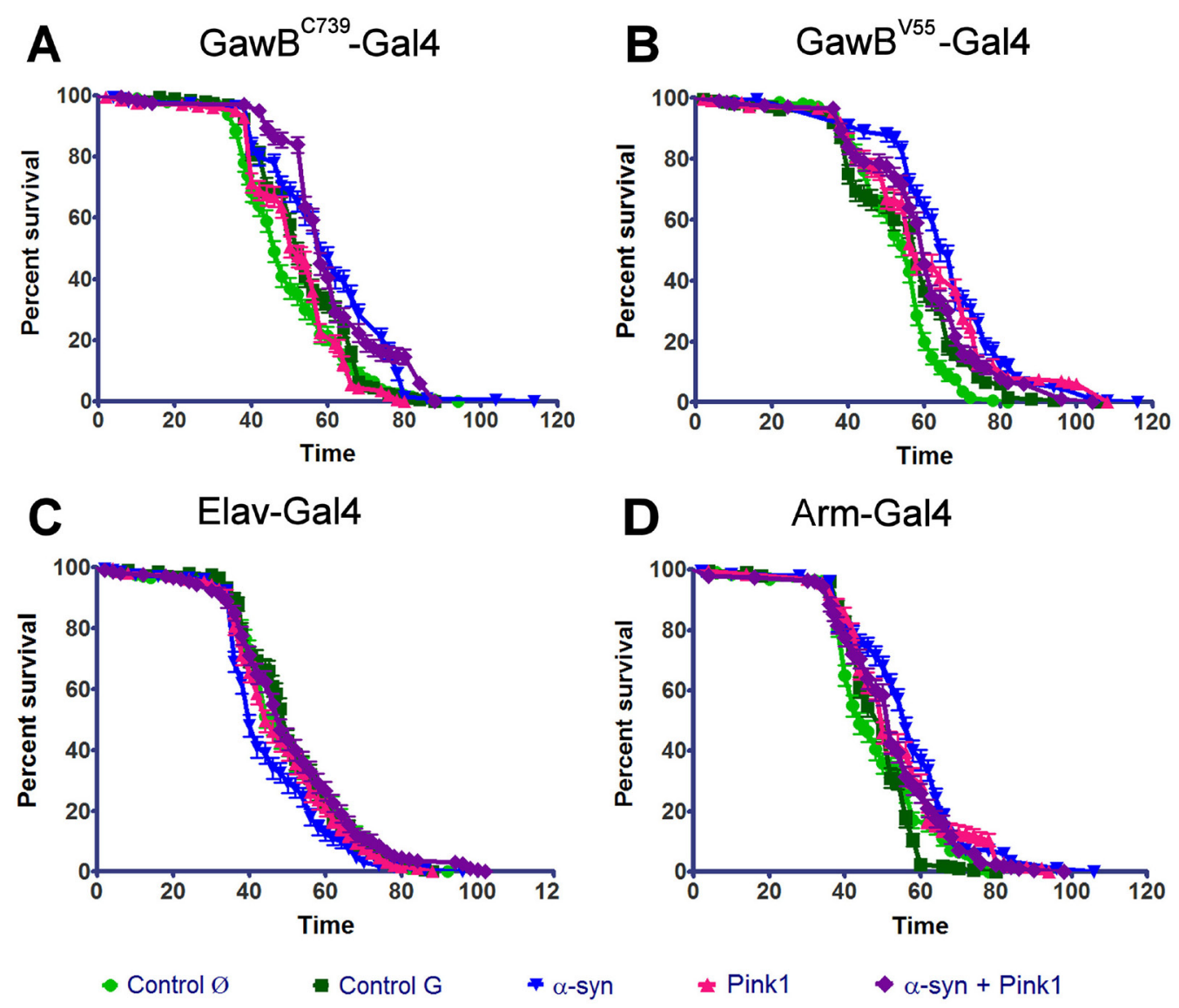

Figure 2. Effect of Pinkl and $\alpha$-synuclein expression on longevity when driven with $G a w B^{C 739}$-Gal4 (A), GawB ${ }^{V 55}$ Gal4 (B), Elav-Gal4 (C), and arm-Gal4 (D). Survival curves were compared using the log-rank test. Genotypes expressed include $w^{1118}$ (Control Ø); UAS-GFP/+ (Control G); UAS- $\alpha$-synuclein/+ $(\alpha$-syn); UAS-Pink1/+ (Pink1), and $U A S$ - $\alpha$-synuclein $/+; U A S-P i n k 1 /+(\alpha$-syn + Pink1). Error bars indicate standard error of the mean.

\section{DISCUSSION}

Our findings show that expression of Pinkl with $\alpha$-synuclein has a synergistic effect when driven with the TH-Gal4 transgene, leading to increased longevity in Drosophila. Healthspan, as measured by climbing ability over time, is also enhanced, suggesting an improvement of functional 
aging in these flies, opposed to longevity alone. This protective effect is of interest, as $\alpha$-synuclein is involved in a central pathogenic mechanism for Parkinson disease and has been linked to various aspects of mitochondrial dysfunction (Schapira and Gegg, 2011). Previous studies indicate that the accumulation of $\alpha$-synuclein in the mitochondria of mammalian dopaminergic neurons leads to reduced mitochondrial complex I activity and increased production of reactive oxygen species (ROS) (Devi et al., 2008; Liu et al., 2009). It is important to note that ROS also act as signaling molecules, and can be involved in a number of pro-survival pathways, including regulation of autophagy (Scherz-Shouval and Elazar, 2007; Weber and Reichert, 2010). In this respect, $\alpha$-synuclein may be involved in the turnover of mitochondria by autophagy, or mitophagy, acting in unison with Pink1 in a pro-survival role via the removal of defective mitochondria.

The increases in lifespan shown with TH-Gal4 have not been observed when using other drivers, including neuronal (Elav-Gal4, GawB ${ }^{C 739}$-Gal4, GawB $B^{V 55}$-Gal4) and ubiquitous (Arm-Gal4) drivers. Within the cell, tyrosine hydroxylase enzyme catalyzes the conversion of 1-tyrosine to 1-dopa, which is the initial and rate-limiting step in the biosynthesis of catecholamines such as dopamine. These dopaminergic neurons are particularly sensitive, perhaps exacerbated by the metabolic stress created by sustained $\mathrm{Ca}^{2+}$ entry during signaling (Surmeier et al., 2010). Pink1 may have a more pronounced protective effect in this particular cell type, as Pink1 acts through a general protective role but can also directly regulate calcium flux through the mitochondria (Deas et al., 2009). Interestingly, previous study in our laboratory expressing $\alpha$-synuclein and Pink1 using Ddc-Gal4 (Todd and Staveley, 2008) has not shown the synergistic effect seen in this study, using TH-Gal4. Dopa decarboxylase catalyzes the last step of dopamine synthesis, 1-dopa to dopamine, and the last step of serotonin synthesis, 1-tryptophan to serotonin. The discrepancies between results seen when using the TH-Gal4 driver and the Ddc-Gal4 driver are likely due to differing coverage of the dopaminergic neurons. There is growing evidence that although all dopaminergic neuron clusters in the fly brain seem to be targeted by the TH-Gal4 driver, they are not covered equally, and that Ddc-Gal4 likely does not target all dopaminergic neuronal clusters (Yarali and Gerber, 2010). This incomplete overlap of dopaminergic neurons targeted by the TH-Gal4 and Ddc-Gal4 transgenes may indicate that there is a particular dopaminergic cell cluster responsible for the increased lifespan observed in this study. It will be important for future studies to examine the differences between the TH-Gal4 and Ddc-Gal4 drivers with respect to aging and functional longevity.

Our results suggest that increases in $\alpha$-synuclein and Pink1 together may have a synergistic effect, allowing for enhanced protection and increased functional longevity in Dro-

sophila. This may be a result of the upregulation of pro-survival mechanisms via Pink1, in response to an increase in ROS signaling due to $\alpha$-synuclein overexpression. The restriction of these results to $\mathrm{TH}$-Gal4-expressing cells likely reflects the existence of a dopaminergic cell cluster that is particularly sensitive to changes in Pink1 and $\alpha$-synuclein expression. Moreover, the results indicate the need for future examination of this particular cell population, where findings may shift therapeutic efforts towards a particular dopaminergic cluster.

\section{ACKNOWLEDGMENTS}

Research supported by the Natural Sciences and Engineering Research Council of Canada (NSERC) Discovery Grants, the Parkinson Society Canada Friedman Pilot Project Grants (BES) and the School of Graduate Studies at Memorial University of Newfoundland (AMT). 


\section{REFERENCES}

Clark IE, Dodson MW, Jiang C, Cao JH, et al. (2006). Drosophila pink1 is required for mitochondrial function and interacts genetically with parkin. Nature 441: 1162-1166.

Deas E, Plun-Favreau H and Wood NW (2009). PINK1 function in health and disease. EMBO Mol. Med. 1: 152-165.

Devi L, Raghavendran V, Prabhu BM, Avadhani NG, et al. (2008). Mitochondrial import and accumulation of alphasynuclein impair complex I in human dopaminergic neuronal cultures and Parkinson disease brain. J. Biol. Chem. 283: 9089-9100.

Exner N, Treske B, Paquet D, Holmström K, et al. (2007). Loss-of-function of human PINK1 results in mitochondrial pathology and can be rescued by parkin. J. Neurosci. 27: 12413-12418.

Feany MB and Bender WW (2000). A Drosophila model of Parkinson's disease. Nature 404: 394-398.

Friggi-Grelin F, Coulom H, Meller M, Gomez D, et al. (2003). Targeted gene expression in Drosophila dopaminergic cells using regulatory sequences from tyrosine hydroxylase. J. Neurobiol. 54: 618-627.

Haywood AF and Staveley BE (2004). Parkin counteracts symptoms in a Drosophila model of Parkinson's disease. BMC Neurosci. 5: 14.

Lees AJ, Hardy J and Revesz T (2009). Parkinson's disease. Lancet 373: 2055-2066.

Li H, Chaney S, Forte M and Hirsh J (2000). Ectopic G-protein expression in dopamine and serotonin neurons blocks cocaine sensitization in Drosophila melanogaster. Curr. Biol. 10: 211-214.

Liu G, Zhang C, Yin J, Li X, et al. (2009). alpha-Synuclein is differentially expressed in mitochondria from different rat brain regions and dose-dependently down-regulates complex I activity. Neurosci. Lett. 454: 187-192.

Park J, Lee SB, Lee S, Kim Y, et al. (2006). Mitochondrial dysfunction in Drosophila PINK1 mutants is complemented by parkin. Nature 441: 1157-1161.

Schapira AH and Gegg M (2011). Mitochondrial contribution to Parkinson's disease pathogenesis. Parkinsons Dis. 2011: 159160.

Scherz-Shouval R and Elazar Z (2007). ROS, mitochondria and the regulation of autophagy. Trends Cell Biol. 17: 422427.

Staveley BE, Phillips JP and Hilliker AJ (1990). Phenotypic consequences of copper-zinc superoxide dismutase overexpression in Drosophila melanogaster. Genome 33: 867-872.

Surmeier DJ, Guzman JN and Sanchez-Padilla J (2010). Calcium, cellular aging, and selective neuronal vulnerability in Parkinson's disease. Cell Calcium 47: 175-182.

Todd AM and Staveley BE (2004). Novel assay and analysis for measuring climbing ability in Drosophila. Drosoph. Inf. Serv. 87: 101-107.

Todd AM and Staveley BE (2008). Pink1 suppresses alpha-synuclein-induced phenotypes in a Drosophila model of Parkinson's disease. Genome 51: 1040-1046.

Wang HL, Chou AH, Yeh TH, Li AH, et al. (2007). PINK1 mutants associated with recessive Parkinson's disease are defective in inhibiting mitochondrial release of cytochrome c. Neurobiol. Dis. 28: 216-226.

Weber TA and Reichert AS (2010). Impaired quality control of mitochondria: aging from a new perspective. Exp. Gerontol. 45: 503-511.

Whitworth AJ, Wes PD and Pallanck LJ (2006). Drosophila models pioneer a new approach to drug discovery for Parkinson's disease. Drug Discov. Today 11: 119-126.

Yang Y, Gehrke S, Imai Y, Huang Z, et al. (2006). Mitochondrial pathology and muscle and dopaminergic neuron degeneration caused by inactivation of Drosophila Pink1 is rescued by Parkin. Proc. Natl. Acad. Sci. U. S. A. 103: 10793-10798.

Yarali A and Gerber B (2010). A Neurogenetic Dissociation between Punishment-, Reward-, and Relief-Learning in Drosophila. Front. Behav. Neurosci. 4: 189. 